\title{
The Ball Mill Load Measuring algorithm though Grinding tone signal based on GA
}

\author{
${ }^{a}$ Yingmin Yi, ${ }^{\text {H} H a i c h u a n ~ Y a n g, ~}{ }^{\mathbf{b}}$ Lu Sun and ${ }^{\mathrm{C}}$ Xiaoli Liu \\ aShaanxi Key Laboratory of Complex System Control and Intelligent Information Processing, Xi'an \\ University of Technology, Xi'an 710048, China \\ ${ }^{\mathrm{b}}$ Shaanxi Science and Technology Holding Group, Xi'an 710077, China \\ ${ }^{\mathrm{c}}$ Research Institute of Electronic Comprehensive Survey, Ministry of Information Industry, Xi'an \\ Shaanxi 710054, China
}

ayiym@xaut.edu.cn, b157405139 @qq.com

Keywords: Ball mill load; Grinding tone signal; Genetic algorithm; RBF neural network.

\begin{abstract}
For a high energy loss and complex system of ball mill, this paper provide a ball mill load detection method based on genetic algorithm optimizing BP neural network. The effective frequency range of mill sound signal is analyzed. The soft measurement model of mill load based on mill sound signal is built. In order to solve the problem which converge slowly and easily reach minimal value, the global optimization of GA (genetic algorithm) local optimization of BP neural network will be combined to improve the BP neural network. Compare with the detected mill load error generated from existing BP neural network and RBF neural network based on K-means. The experiments results show that the proposed algorithm has better precision.
\end{abstract}

\section{Introduction}

The ball mill is the main equipment of material smashing and its energy consumption occupy $15 \% \sim 25 \%$ of the whole company. So the measurement of ball mill load has a important significance of production at the aspect of ensuring safety, improve efficiency and reducing energy consumption.

Because the ball mill load was influenced by many factors with complex relationships, the detection became difficult which is still a technological difficulties for a long time. For this, oversea and domestic scientists have done a lot of researches [1]. So far, the main method to measure the ball mill load are direct and indirect methods. The direct method has been put forward in the international mineral processing congress at 1973. The direct method use pole to measure the height of ore pulp liquid level to get the load. It cannot be popularized since the problems of pole damage and installation site haven't been solved. The indirect method include the audio frequency method, the vibration signal method, the active power method, the air pressure method and so on. Audio frequency method has the advantages of low freight, easy control and simple structure. But the recent adopted BP neural network and RBF neural network are not accurate enough at the predicting of mill load. The vibration signal method usually use correlation analysis and spectral analysis. But the instability of the electrified wire netting frequency lead to detection instability. The active power method is not easy to be affected by surrounding. But the disadvantage is that the ball mill power is affected by steel ball load, the sensitivity of detection signal is low [5]. Seriously affected by the revolving speed of ball mill, elevator abrasion and the granularity distribution inside ball mill, the air pressure method is hardly popularized [6].

Since ball mill is a complex system with time-varying, multivariable, nonlinear and large time 
delay, the mill load status can't be obtained directly through mechanism modeling approach. Artificial neural network provides a new approach for ball mill load to build soft measurement models because it needn't acquire the transcendental knowledge of object, but according to Its input and output to model directly. The commonly used neural network is BP neural network and RBF neural network; BP neural network is multi-layered with back-propagation error and RBF neural network is a forward network with two layers. Compare with BP neural network, there isn't local optimum problem in RBF study algorithm.

In order to solve the problem which converge slowly and easily reach minimal value, the global optimization of GA (genetic algorithm and) local optimization of BP neural network will be combined to improve the BP neural network. This paper provides a mill load detection method based on BP neural network optimized by GA, this method is able to find out the effective range of mill sound and line out the percent corresponding relationship between mill sound's frequency spectrum and mill load after frequency-selecting. According to the measurement model of mill load, mill load can be accurately calculate to realize auto-control and energy-saving and cost-reducing in industry.

\section{A Ball Bill Load Detection Method Based on Genetic Algorithm Optimizing BP Neural Network}

In the paper, the structure of designed soft measurement model of mill load detection is shown in Fig.1. Mill load detection method used mill sound signal include: mill sound signal collection, mill sound signal processing, effective frequency band selection, mill sound calibrating and soft measurement output.

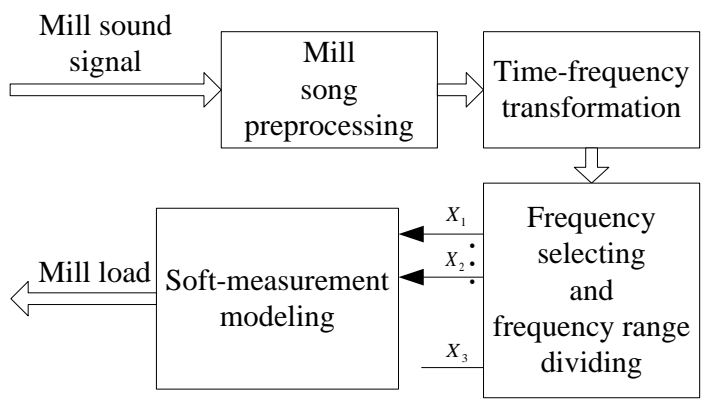

Fig. 1 The soft-sensing model structure of ball mill load measuring

\subsection{The Step of Ball Mill Load Detection Used Mill Sound:}

Step 1: collect the initiative mill sound and the terminated mill of the ball mill;

Step 2: Process the mill sound off line;

a. To avoid the interference between the initiative mill sound and terminated mill sound at the moment of beginning and ending, break off both ends of mill sound;

b. Process the initiative mill sound and terminated mill sound as a constant length after breaking off both ends to get multiple information of data segments. Use Welch Method to add each data segments Hamming Window, the expression of Hamming Window as equation (1);

$w(n)\left\{\begin{array}{cc}0.54-0.46 \cos \left(\frac{2 \pi n}{L-1}\right) & 0 \leq n \leq 1 \\ 0 & \text { other }\end{array}\right.$

c. Then conduct fast flourier transformation on the windowed date segments, take the square of 
amplitude of each transformed results to divide the number of transformation point as a estimated value of power spectrum estimation; turn this value to sound pressure level.

Step 3: Select the effective frequency band of mill sound;

Mark off 4-8 kinds mill load from initiative mill sound and terminated mill sound, plot out the tendency charts of mill sound intensity of mill load changing over frequency; Select the frequency of original mill sound data according to effective frequency band to get the selected mill sound data;

Step 4: Calibrate the mill load data;

Step 5: Build a model based on genetic algorithm optimizing BP neural network and forecast the mill load to get the result of ball mill load detection.

\subsection{The Design of Genetic Algorithm Optimizing BP Neural Network}

Genetic algorithm optimizing BP neural network can be divide to three parts: the structure of neural network, GA optimizing and BP neural network forecast. The algorithm flow chart is shown in Fig.2. Among them, the structure of BP neural network can be determined according to the number of IO parameters in fitted function, and then determine the individual encoding length of GA. GA optimizing is that using genetic algorithm to optimizing the weight and threshold of BP neural network. The individual encoding, in GA population, is constituted by all the weight and threshold of BP neural network comply with a kind of rule, use defined fitness function to work out the fitness value of each individuals. GA process the individuals that corresponding to optimal fitness value through selecting, crossing and varying operator. Evaluate the optimal individuals, get from GA, to the weight and threshold of BP network, then the network forecast the output of function after training.

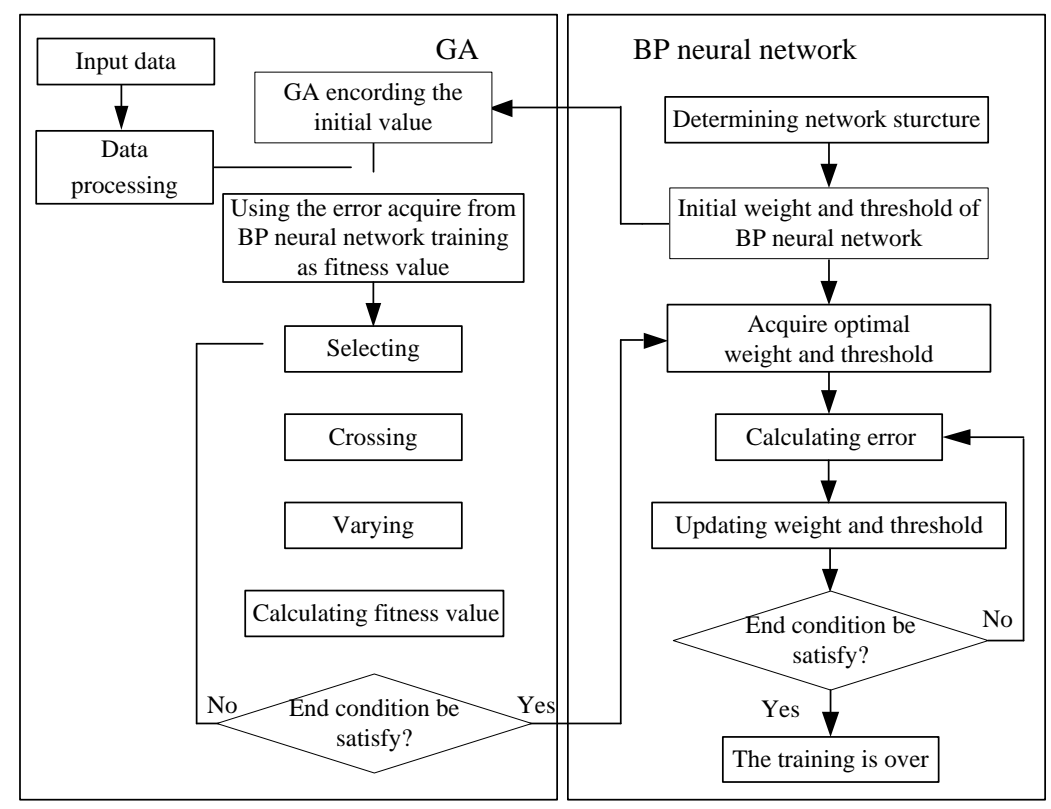

Fig.2The flowchart of GA to optimize BP neural network

GA optimizing neural network is that evaluate the optimal original weight and threshold, get from GA, to BP neural network to make it get a better network model, and then forecast the output more well. The realizing step of GA optimizing BP neural network as follow:

Step 1: Population encoding

Individual encoding text use real number encoding to get a bigger searching space. Each individual are encoded to a real number string. The real number string is constituted of the weight from input layer to concealed layer, the threshold of concealed layer, the weight from concealed layer to output layer and threshold of output layer. 
Step 2: Work out the fitness function

According to the original weight and threshold of BP neural network of individual, after using training sample data to train BP neural network, forecast the output of network, then regard the error between expected and predicted output as individual fitness value F. The computing method is

$$
F=k\left(\sum_{i=1}^{n} a b s\left(y_{i}-o_{i}\right)\right)
$$

In the equation, $n$ is node number of network output layer; $o_{i}$ is the predicted output of the node $i ; y_{i}$ is the expected output of the node $i ; k$ is a coefficient.

\section{Step 3: Selection}

There are may methods of genetic algorithm selection, such as random traversal sampling and roulette method. Roulette method is selected in this paper. Roulette method select objects according to fitness proportion, the selected probability $p_{i}$ of each individuals is $p_{i}=\frac{f_{i}}{\sum_{j=1}^{N} f_{i}}$.

In the equation, $f_{i}$ is the fitness value of individual $i$, because the fitness value is more small more good, the fitness value should be differentiated before selecting individuals; $k$ is a coefficient; $N$ is the number of individuals in this population.

Step 4: Crossing

Since individual encoding use real number encoding, according to the method of real number crossing, the crossing method of the $k$-th chromosome $a_{k}$ and the $l$-th

$a_{i}$ in the $j$ place as follow:

$$
\left.\begin{array}{l}
a_{k j}=a_{k j}(1-b)+a_{l j} b \\
a_{l j}=a_{l j}(1-b)+a_{k j} b
\end{array}\right\}
$$

In the equation, $b$ is a random number among $[0,1]$.

Step 5: Varying

Select the gene $a_{i j}$ of the $i$-th individual to vary, the method of variation as follow:

$$
a_{i j}= \begin{cases}a_{i j}+\left(a_{i j}-a_{\max }\right) \times f(g) & r \geq 0.5 \\ a_{i j}+\left(a_{\min }-a_{i j}\right) \times f(g) & r<0.5\end{cases}
$$

In the equation, $f(g)=r_{2}\left(1-g / G_{\max }\right) ; a_{\max }$ is the upper bound of gene $a_{i j} ; a_{\min }$ is the lower bound of gene $a_{i j} ; g$ is the current evolution time; $r_{2}$ is a random number; $r$ is a random number among [0,1]; $G_{\max }$ is the maximal iteration time.

Step 6: Train the optimized weight and threshold in BP network to forecast.

\section{Experiment and Analysis}

The mill sound in this experiment is a actual working signal at a mine factory, the mill object is $\phi 3.6 \times 4 \mathrm{~m}$ middle-sized mill, the diameter of metal balls is about $70 \mathrm{~mm}$, the ore is 
porphyry-disseminated molybdenum ore, the revolving speed is $18 \sim 22 \mathrm{r} / \mathrm{min}$; It used 16 bit single channel sampling with the frequence of $44100 \mathrm{~Hz}$. It mainly sample signal when ball mill was turning from normal working to stop and from launch to normal working. Used recording pen to record real-time sound near the noise sensor of the experimental ball mill.

\subsection{The Verification of The Ball Mill Load Detection Method Based on Genetic Algorithm Optimizing BP Neural Network}

In order to observe the tendencies of frequence change over mill load change, the mill sound intensity change tendency of four types of mill load states over frequency is shown in Fig.3.

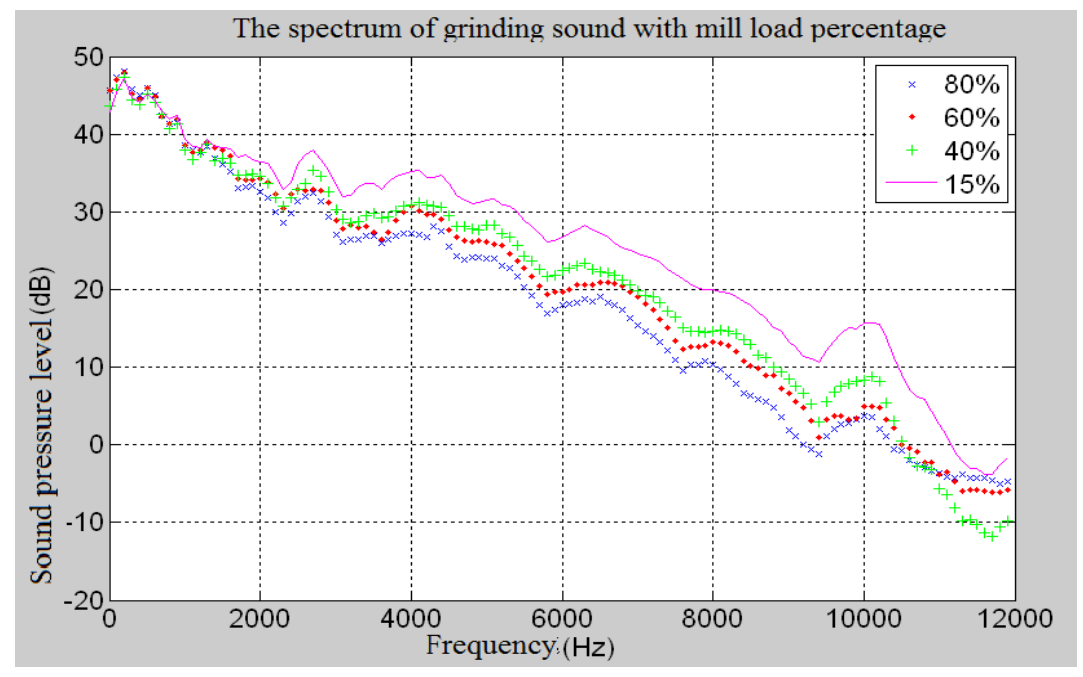

Fig.3 The spectrum of grinding sound with mill load percentage

It can be seen shown in Fig.3 that in the same frequency, mill sound energy increased over mill load decreasing and this phenomenon is more obvious in high frequency than the low which conform to the mill theory analyzed before. It can be found from the figure that below $1500 \mathrm{~Hz}$, the change of mill sound energy is not obvious over mill load due to the interferential noise during the period of working, these noise lead to a length of frequency of mill sound signal independent of load change. Over the frequency of $10 \mathrm{kHz}$, compare with the high energy in middle and low frequency band, the amplitude is too small to be considered.

According to the designed ball mill load detection method based on genetic algorithm optimizing BP neural network, the structure of BP neural network is determined as 8-7-1, eight nodes for input layer, seven for hide layer and one for output layer, a total of $8 * 7+7 * 1=63$ weights and $7+1=8$ thresholds, the individual encoding length of $G A$ is $63+8=71$. Select 79 groups of data from the known 100 groups for training and 21 groups for testing. The selection of population scale as follow: Use real number encoding at population initialization with the range of $[-1,1]$. Fitness function: The sum of error absolute value is considered between expected and predicted output as the individual fitness value. The maximal evolutionary generations: maxgen=50. Crossing probability: pcross=0.4. Variation probability: pmutation $=0.09$.

Tab.1 Select the size of the population

\begin{tabular}{|c|c|c|}
\hline Population scale & Training error & Training time (s) \\
\hline 10 & 38.0292 & 44.8186 \\
\hline 15 & 26.4447 & 69.6549 \\
\hline 20 & 8.0864 & 81.8541 \\
\hline 25 & 5.8809 & 156.1202 \\
\hline
\end{tabular}


It can be shown as Tab.1, with the increasing of population scale, the training error reduces and the training time increases; Comprehensive consideration to determine the population scale in this paper: sizepop=20.

\subsection{Error analysis of experimental results}

The predicted absolute output error of this three methods is shown as Fig.4. It can be shown from the figure that the output error of the three models below 6\% during first 12 minutes, genetic algorithm optimizing BP neural network achieves the best effect; In 13 minutes, the effect of the three models becomes worse due to the immeasurability of mill load, there is error when calibrating the mill load data.

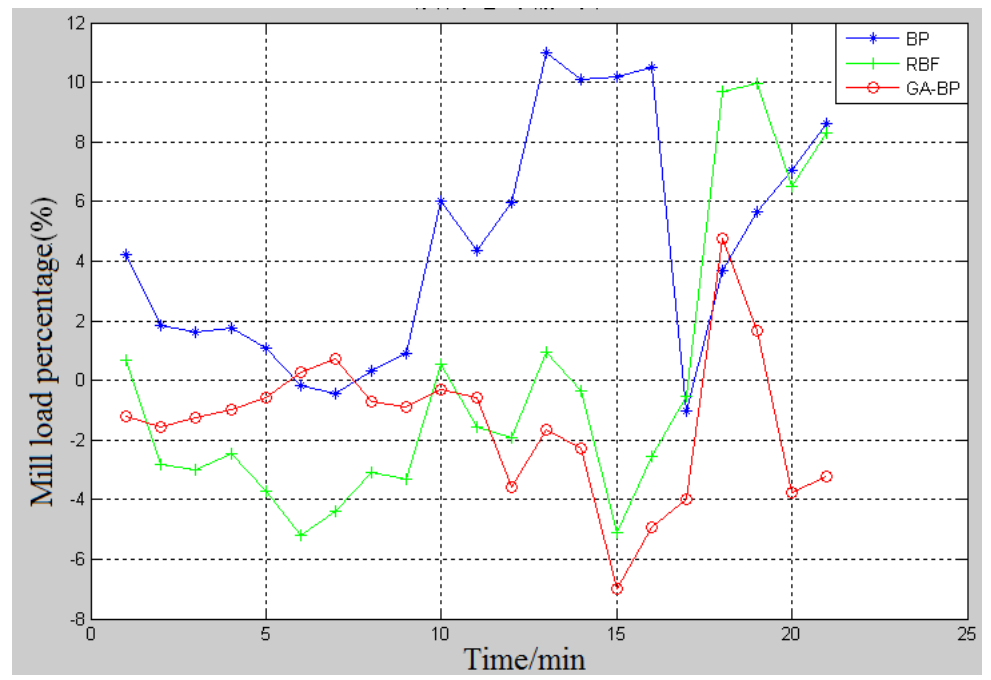

Fig.4 The predict output error graph of three models

The analysis results is shown as Tab.2.

Tab.2 Comparing results of three models

\begin{tabular}{|c|c|c|c|}
\hline Item compared & BP neural network & RBF neural network & $\begin{array}{l}\text { GA optimizing } \\
\text { BP neural network }\end{array}$ \\
\hline $\begin{array}{c}\text { Mean square } \\
\text { error }\end{array}$ & 21.2958 & 21.3493 & 8.0864 \\
\hline $\begin{array}{c}\text { Maximum } \\
\text { absolute error }\end{array}$ & 9.8159 & 10.5481 & 7.0111 \\
\hline $\begin{array}{c}\text { Mean absolute } \\
\text { error }\end{array}$ & 3.6217 & 3.4782 & 2.1936 \\
\hline $\begin{array}{c}\text { Average } \\
\text { relative error }\end{array}$ & 0.1051 & 0.1625 & 0.0793 \\
\hline $\begin{array}{c}\text { Mean square } \\
\text { relative error }\end{array}$ & 0.1623 & 0.3127 & 0.1528 \\
\hline $\begin{array}{c}\text { Iterations } \\
\text { of BP network) }\end{array}$ & $\begin{array}{c}3 \text { (cluster times of } \\
\text { K-means) }\end{array}$ & $\begin{array}{c}3 \text { (Training times } \\
\text { of BP network) }\end{array}$ \\
\hline Training time (s) & 1.343634 & 0.1334 & 81.854120 \\
\hline
\end{tabular}

Through Tab.2, BP network is easy to fall into local minimum, so it can't reach the global minimum that slows down the convergence speed; Because of the only best approximation of RBF network based on K-means, both the training accuracy and the convergence speed are more superior than BP network, but it entirely dependents on the selected data sample; The optimal original weight and threshold, acquired from optimized BP network through GA, make the global 
optimization is easy to be achieved and enhance the network forecast accuracy and convergence speed.

\section{Conclusion}

For such a high energy loss and complex system of ball mill, this paper provide a ball mill load detection method based on genetic algorithm optimizing BP neural network. Through the analysis of actual mill sound signal of medium-sized ball mill at a mine, this method can analyze the effective frequency range of mill sound signal and build the soft measurement model of mill load based on mill sound signal. Compare with the detected mill load error generated from existing BP neural network and RBF neural network based on K-means, the availability of the proposed algorithm.

\section{Acknowledgement}

In this paper, the research was sponsored by the Nature Science Foundation of Shaanxi Province (Project No. 16JS069) and Shaanxi Synergy Innovation Center (Project No. 304-210891702) and Xi'an science and technology plan(Project No. 2017080CG/RC043(XALG005)) .

\section{References}

[1] D. Hodouina, S.-L.J.ams.a-Jounelab, M.T.Carvalhoc, L.Berghd. State of the art and challenges in mineral processing control[J]. Control Engineering Practice , 2001,9(11):995-1005.

[2] Tang Jian, Zhao Lijie, Yue Heng, Chai Tianyou. Present Statues and Future Development of Detection Method for Mill load[J].Control Engineering of China,2010,09:17-5.

[3] Wu Zhimin, Hu Xuanli, Huang Xieqing. On the Construction and Operation of Distributive Resources of Design Knowlege[J] China Mechanical Engineering, 1998,9(2):30-33.

[4] Tang Jian, Chai Tianyou, Zhao Lijie, Yue Heng. Ensemble Modeling for Parameters of Ball-Mill Load in Gringind Process Based on Frequency Spectrum of Shell Vibration[J].Control Theory \& Applications, 2012,29(2):183-191.

[5] Mao Yiping, Chen Bingchen, Gao Jisen. Factor Affecting Ball Mill's Active Power and Grinding Efficiency[J]. Mining and Metallurgical Engineering,2000, 20(4):48-50.

[6] Kolacz J. Measurement system of the mill charge in grinding ball mill circuits[J]. Minerals Engineering, 1997,10(12):1329-1338.

[7] Ge Zhihui, Zeng Wennan, Zhao Baokun. Summing-up of Automatic Delecting and Automation in Mineral Processing[J]. China Mine Engineering,2006,35(6): 37-42.

[8] Wang Heng, Jia Minping, Chen Zuoliang. Method of Optimize Control for Ball Mill Based on Soft Sensor[J]. Control Engineering of China,2011, 18(5):762-766.

[9] F.Flament, J.Thibault, D.Hodouin. Neural Network based control of mineral grinding plants[J]. Mineral Engineering,1993,6(3): 235-248.

[10]Gao Xiang, Ren Guochun, Chen Jin, Ding Guoru. Support Vector Regression-based Spectrum Prediction Under Quick-Changing Channel Occupancy [J]. Journal of Signal Processing,2014,30(3):289-297. 